DOI: $10.17957 / \mathrm{IJAB} / 15.1795$

http://www.fspublishers.org

\title{
SIX6 Shows High Divergence in Fusarium oxysporum f. sp. cubense TR4
}

\author{
Nadya Farah ${ }^{1,2}$, Mohammad Bahrelfi Belaffif ${ }^{3}$, I. Nyoman P. Aryantha ${ }^{1}$ and Rizkita Rachmi Esyanti ${ }^{1 *}$ \\ ${ }^{1}$ School of Life Sciences and Technology, Institut Teknologi Bandung, Bandung 40132, Indonesia \\ ${ }^{2}$ Department of Biology, Indonesia Defense University, Bogor 16810, Indonesia \\ ${ }^{3}$ Agria Analitika Indonesia, Jakarta 12560, Indonesia \\ *For correspondence: rizkita@sith.itb.ac.id \\ Received 25 December 2020; Accepted 20 March 2021; Published 10 May 2021
}

\begin{abstract}
Secreted fungal effector proteins and their host targets are good examples to understand the mechanism of host-pathogen coevolution with genes involved in the interaction undergoing positive selection. SIX genes (secreted in xylem) are obtained via horizontal transfer and can be found within the formae speciales of Fusarium oxysporum. SIX6 and SIX9 of F. oxysporum $\mathrm{f}$. spp. cubense $(F o c)$ are predicted to play a role as effectors. However, their involvement in the pathogenicity of $F o c$ in banana plants has not been determined yet. In the susceptible banana cultivar, we found that the SIX6 and SIX9 genes of Foc TR4 were highly expressed in roots, but not in corms or leaves. The host, however, expressed the pathogenesis-related (PR) genes, $P R-1$ and PR-3, in corms earlier than in the roots. Phylogenetic analysis on SIX6 and SIX9 genes of $F$. oxysporum has revealed the separation of SIX6 and SIX9 of Foc from other formae speciales. This leads to detecting genes under positive selection using the ratio nonsynonymous to synonymous substitution rates $(\mathrm{Ka} / \mathrm{Ks})$. SIX6 of Foc showed an increase in diversity, but insufficient to drive positive selection. Conversely, SIX9 of Foc showed no divergence in the dN/dS ratio distribution, indicating purifying selection. (C) 2021 Friends Science Publishers
\end{abstract}

Keywords: Effector evolution; Ka/Ks ratio; Positive selection; Purifying selection; SIX effectors

\section{Introduction}

The never-ending battle between pathogens and hosts leads to a co-evolutionary arms race where both evolve to counteract each other (Derbyshire 2020). Hosts develop strategies to recognize pathogens and escape infections whereas pathogens develop ways to avoid host recognition and escape host defenses. The dynamics between secreted fungal effector proteins and their host targets are good examples in understanding the mechanism of host-pathogen co-evolution with genes involved in the interaction undergo positive selection (Presti et al. 2015). A successful pathogen must be able to maintain the ability to avoid host recognition but still virulent in the process. This will determine infectivity and host specialization. In order to do this, pathogens will have to pass a series of gene modifications, changes in the expression of existing effector genes, or even generate new effectors (Presti et al. 2015).

Generally, effectors are modular proteins. They contain signal peptides that are relatively small in size, rich in Cysteine residues, and do not have similarities with known proteins (Stergiopoulos and Wit 2009; Sonah et al. 2016; Dalio et al. 2018). In host cells, effectors may suppress host defense systems or deceive host cells to accommodate further infection and colonization (Dodds et al. 2009). Fungal pathogens have developed the ability to deliver effectors inside the host cytoplasm as well as in the extracellular space, thus classified as cytoplasmic and apoplastic effectors, respectively (Wang et al. 2017).

Banana is the fourth most important export commodity worldwide after rice, wheat, and corn (FAO 2020). However, the sustainability of banana production worldwide is threatened by pests and diseases such as Fusarium wilt caused by Fusarium oxysporum f. spp. cubense (Dita et al. 2018). To counteract this pathogen, molecular studies conducted to identify resistance genes expressed by the host cells and genes involved in virulence or pathogenicity are urgently needed. Until recently, genomic, transcriptomic proteomics analyses have been conducted in Foc TR4 (Guo et al. 2014; Sun et al. 2014) and also on banana cultivars that are susceptible and/or resistant to Foc TR4 (Li et al. 2012; Bai et al. 2013; Sun et al. 2019; Zhang et al. 2019). These studies are crucial in order to develop effective methods to manage the pathogen while being wary in the emergence of resistance in banana plants. Although host adaptation and specificity within formae speciales of diverse pathogenic fungus, including $F$. oxysporum, have been studied extensively ( $\mathrm{Li}$ et al. 2020), the evolutionary origin of the host specificity gene is still undetermined. Ma et al. (2010) revealed four lineage-specific

To cite this paper: Farah N, MB Belaffif, INP Aryantha, RR Esyanti (2021). SIX6 shows high divergence in Fusarium oxysporum f. sp. cubense TR4. Intl J Agric Biol 25:1331-1338 
chromosomes in $F$. oxysporum, one of which is the $2-\mathrm{Mb}$ chromosome 14 of $F$. oxysporum f. spp. lycopersici ( Fol). Chromosome 14 consists of genes encoding secreted effectors such as the SIX genes, of which many are involved in pathogenicity. It is suggested that the pathogenicity of nonpathogenic $F$. oxysporum strain towards tomato is acquired by the acquisition of Fol chromosome 14 by horizontal chromosomal transfer (Mehrabi et al. 2011).

The SIX effectors initially found in Fol that infects tomato were SIX1 (Rep et al. 2004), SIX2, SIX3 and SIX4 (Houterman et al. 2007), SIX5, SIX6 and SIX7 (Ma et al. 2010). In tomato, $S I X 1$ (also known as $A v r 3$ ) is required for Fol virulence (Rep et al. 2005) and I-3-mediated resistance (I for immunity) (Rep et al. 2004). SIXI was found consistently in Foc strains, with 3 homologs found in TR4 (SIX1a, b and c) (Widinugraheni et al. 2018). SIX1 is also known to be involved in Foc virulence in Cavendish (Widinugraheni et al. 2018). SIX4 (also known as Avrl) plays a role in I-1-mediated resistance but suppresses the I2 and I-3-mediated resistance (Houterman et al. 2008). Similar to SIX1, SIX3 (also known as Avr2) is required for Fol virulence in susceptible hosts and triggered resistance in tomato plants containing the $I-2$ resistance gene (Houterman et al. 2009). Furthermore, SIX8 was reported to be involved in the virulence of Foc TR4 into Cavendish (An et al. 2019). Up to now, a total of 14 effectors have been identified in bananas (Czislowski et al. 2018) and SIX gene homologous have been found in $F$. oxysporum infecting other plants, such as tomato, date palm, melon, passionfruit, pea, watermelon, common bean, and cucumber (Thatcher et al. 2012; Laurence et al. 2015). SIX6 and SIX9 of Foc have been examined in numerous studies (Czislowski et al. 2018; An et al. 2019). However, their role in pathogenicity in banana plants has not been determined. In this study, we aimed to provide new evidence to support the hypothesis that SIX6 and SIX9 of Foc could play a role as effectors.

\section{Materials and Methods}

\section{Plant materials and pathogen inoculation}

Cavendish "Grand Nain" plantlets were propagated in Murashige and Skoog (MS) media containing $2.5 \mathrm{ppm}$ of benzyl amino purine (BAP). Plantlets with 3-5 leaves were selected for inoculation with Foc TR4 isolated from infected banana cv. Bading kayu susu Banana cultivars were grown at room temperature with a $16 \mathrm{~h}$ day (approximately $200 \mu$ mol m$~^{-2} \mathrm{~s}^{-1}$ light intensity) $/ 8 \mathrm{~h}$ night cycle. Foc isolate was grown in Potato Dextrose Agar medium for 7 days at room temperature and prepared as $10^{6}$ spore $\mathrm{mL}^{-1}$ suspensions in $0.85 \% \mathrm{NaCl}$. Plantlets were acclimatized 2 days prior to infection in MS and inoculated with $1 \mathrm{~mL}$ of $F O c$ suspension. Samples of roots, corms, and leaves of infected bananas were collected 3, 6, 9 and 14 days post-infection. Each time point is consisted of at least a collection of 2-3 plantlets.

\section{RNA extraction and quantitative real-time PCR}

Total RNAs were isolated from the roots, corms, and leaves of the infected banana cv. Cavendish 3, 6, 9 and 14 days post-infection as described by Cordeiro et al. (2008). Firststrand cDNA synthesis was performed with 1 gram of total RNA employing the iScript cDNA synthesis kit according to the manufacturer's instruction (Biorad, California, USA). The expression of SIX6, SIX9, PR-1 and PR-3 genes and $G A P D H$ reference gene (Li et al. 2015) were quantified using the GoTaq ${ }^{\circledR}$ qPCR master mix (Promega, Wisconsin, USA) in QuantStudio 1 Real-Time PCR System (Applied Biosystem, California, USA) and presented as relative expression ( $S I X 6$ and $S I X 9$ ) and normalized expression (PR-1 and PR-3) (Livak and Schmittgen 2001). Three replicates of each sample were analyzed to ensure reproducibility and reliability.

\section{Bioinfomatics tools for in silico study}

The signal peptide cleavage site of SIX6 and SIX9 homologs was determined using the SignalP (http://www.cbs.dtu.dk/services/SignalP-4.1/). The phylogenetic tree of SIX6 and SIX9 in formae speciales of $F$. oxysporum was generated using the IQ-TREE (iqtree.cibiv.univie.ac.at). Putative 3D structures of SIX6 and SIX9 were generated using trRosetta (https://yanglab.nankai.edu.cn/trRosetta/). SNPs (singlenucleotide polymorphisms) were plotted into the putative 3D structures of SIX6 and SIX9 using PyMOL. The Ka/Ks ratio was calculated to identify the site-specific positive selection and purifying selection of SIX6 and SIX9 using Selecton (http://selecton.tau.ac.il/index.html). Pairwise Ka ( $\mathrm{dN}$; rate of nonsynonymous mutation) and $\mathrm{Ks}(\mathrm{dS}$; rate of synonymous mutation) of SIX6 and SIX9 genes was analyzed by running pairwise comparisons between CoDing Sequence (CDS) of SIX6 and SIX9 from different formae speciales using SNAP (Korber 2000) (https://www.hiv.lanl.gov/content/sequence/SNAP/SNA P.html).

\section{Results}

\section{SIX6 and SIX9 highly expressed in roots of Foc-infected bananas}

SIX6 and SIX9 genes were highly expressed in roots of infected bananas, but not in corms or the leaves (Fig. 1A and B). In Cavendish roots, the expression of the SIX6 gene was elevated as high as 1.03 at 6 days post-infection (dpi), whereas SIX9 was 1.07 at 9 dpi. The expression of pathogenesis-related $(P R)$ genes $P R-1$ and $P R-3$ in susceptible cultivar Cavendish was examined during infection. $P R-1$ was expressed early in the corms, 3 and 6 dpi, with 8.67 and 8.75-fold expression, respectively (Fig. 1C). In roots, the highest expression was reached at 9 dpi 

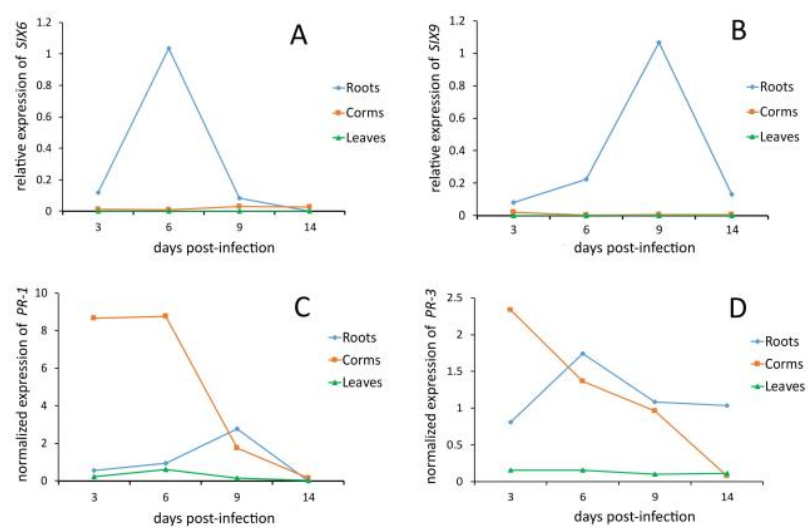

Fig. 1: Gene expression of SIX6, SIX9, $P R-1$ and $P R-3$ in Cavendish banana after infection with Foc TR4. (A) Relative expression of SIX6 and (B) SIX9 gene in roots, corms and leaves of Cavendish 3, 6, 9, and 14 days after infection (dpi) with Foc TR4. (C) Normalized expression of $P R-1$ and (D) $P R-3$ gene in roots, corms and leaves of Cavendish 3, 6, 9, and 14 dpi with $F o c$ TR4. The GAPDH was used as a reference gene

with 1.74-fold expression. Similar to $P R-1, P R-3$ was expressed in the corms 3 dpi with 2.34-fold expression whereas in roots the highest expression was reached at 6 dpi with 1.74-fold expression (Fig. 1D). The expression of both $S I X$ and $P R$ genes was considerably low in the leaves.

\section{$S I X 6$ and $S I X 9$ are predicted to be effectors}

Both proteins contained a signal peptide that cleaved between amino acids in positions 16 and 17 for SIX6 and

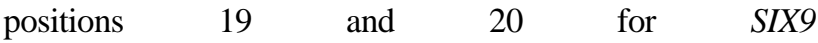
(http://www.cbs.dtu.dk/services/SignalP-4.1/). Homologs of SIX6 and SIX 9 were also cleaved at the same site (Fig. 2 and 3 , respectively). Eight and six Cysteine (C) residues were identified to be conserved among all formae speciales of $F$. oxysporum in SIX6 and SIX9, respectively (Fig. 2 and 3).

\section{SIX6 and SIXY of Foc are polymorphic compared to other formae speciales}

Foc SIX6 and SIX9 shared 51.61 and $44.07 \%$ homology to other formae speciales, respectively (Fig. 2 and 3). Phylogenetic tree of SIX6 and SIX9 genes (Fig. 4 and 5) showed the separation of Foc SIX6 and SIX9 from Fol and other formae speciales. The Foc SIX6 is in a different clade from all other formae speciales, whereas the Foc SIX9 is in the same group with the SIX9 of F. oxysporum f. spp. pisi (accession number MT710731.1), but in the different clade with Fol and the other formae speciales. This indicates high polymorphisms in the sequences of Foc SIX6 and SIX9 genes. The resulted amino acid sequences showed high variations in the signal peptides of Foc SIX6 and SIX9, with 43.75 and $63.16 \%$ respectively, polymorphic to other formae speciales (Fig. 2 and 3 ).

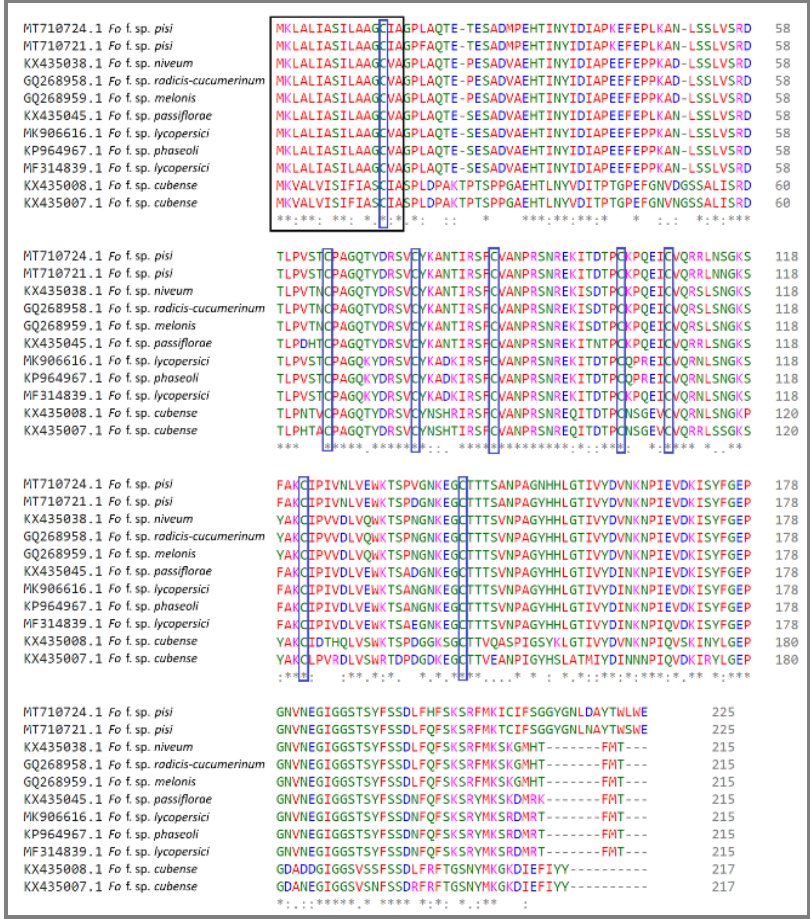

Fig. 2: Alignment of SIX6 in eight formae speciales of $F$. oxysporum, namely f. spp. pisi, f. spp. niveum, f. spp. radiciscucumerinum, f. spp. melonis, f. spp. passiflorae, f. spp. lycopersici, f. spp. phaseoli, and f. spp. cubense. Signal peptide is boxed in black, Cysteine (C) residues in blue box. Sequences were aligned using EMBL Multiple Alignment

\begin{tabular}{|c|c|c|}
\hline 710731.1 fo f. sp. pisi & 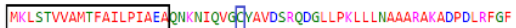 & 60 \\
\hline KX435017.1 Fo f. sp. cubense & MKLLSTVVAMTFAILPIAEAWNKNIOVGdYAVDSRODGLLPKLLLLNAAARAKADPDLRFGF & 60 \\
\hline KX435016.1 Fo f. sp. cubense & INIQVGgVAVDSRQDGLLPKLLLNAAARGKADPDLRFGF & 60 \\
\hline KX435015.1 Fo f. sp. cubense & MKLSAVAAMAFATFHIAEA WNKNIOVGdVAVDSRODGLLPKLLLLNSDARAKADPDLRFGF & 60 \\
\hline MK9e6659. 1 Fo f. sp. Iycopersici & MKLLAVVATALAVFSTAEART - -TQVGQRALDTKNDGLLTELLLNPSARGAADPDLRYGF & 58 \\
\hline KX435847.1 Fo f. sp. possifforae & IIKLLAVVATALAVFSTAEART- - TQVGGRALDTKNDGLLTELLLNPSARGAADPDLRYGF & 58 \\
\hline KX435041.1 Fo f. sp. niveum & 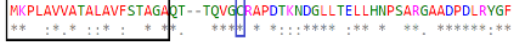 & 58 \\
\hline MT710731.1 Fo f. sp. pisi & 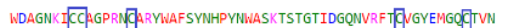 & 18 \\
\hline KX435017.1 Fo f. sp. cut & 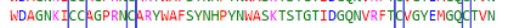 & 118 \\
\hline KX435016.1 Fo f. sp. cubense & WDAGNKICCAGPRNAGRYWAFTYNHPYNWASKTSTGTIDGQNVRFTIVUGYEMGQCTVN & 118 \\
\hline KX435015.1 Fo f. sp. cubense & 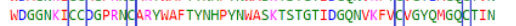 & 118 \\
\hline MK906659. 1 Fo f. sp. Iycopersici & YTFSYNHPYPWAYRQRRGTIRGQQFDFACWNWRTGACK & 114 \\
\hline KX435047.1 fo f. sp. passifforae & WDAKWRRCCUEONID RYYTF SYNHPYPWSYRQRTGTIRRQQFDFACWNUHTGACK & 114 \\
\hline KX435041.1 Fo f. sp. niveum & 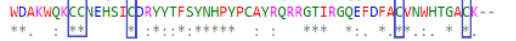 & 114 \\
\hline
\end{tabular}

Fig. 3: Alignment of SIX9 in five formae speciales of $F$. oxysporum, namely f. spp. pisi, f. spp. cubense, f. spp. lycopersici, f. spp. passiflorae and f. spp. niveum. Signal peptide is boxed in black, Cysteine (C) residues in blue box. Sequences were aligned using EMBL Multiple Alignment

When we compared the sequences of Foc SIX6 and SIX9 obtained from the genebank (KX435008.1, and KX435007.1 for SIX6, and KX435015.1, KX435016.1 and KX435017.1 for SIX9) with other SIX6 and SIX9 sequences from different formae speciales, we found 14.75 and $13.56 \%$ polymorphisms in the Foc SIX6 and SIX9 amino acid sequences, respectively. The plotted polymorphisms in the putative 3D structure of Foc SIX6 and SIX9 can be seen in Fig. 6A and B. The polymorphic residues in SIX6 are concentrated in the half downstream of the N-terminus, but the signal peptide residues are conserved. The SIX9, 


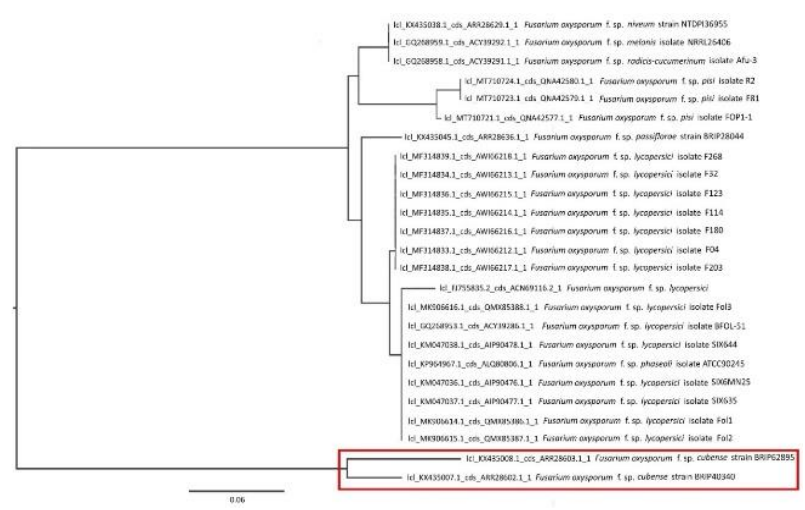

Fig. 4: Phylogenetic tree of SIX6 in formae speciales of $F$. oxysporum. The tree was generated using IQ-TREE (iqtree.cibiv.univie.ac.at)

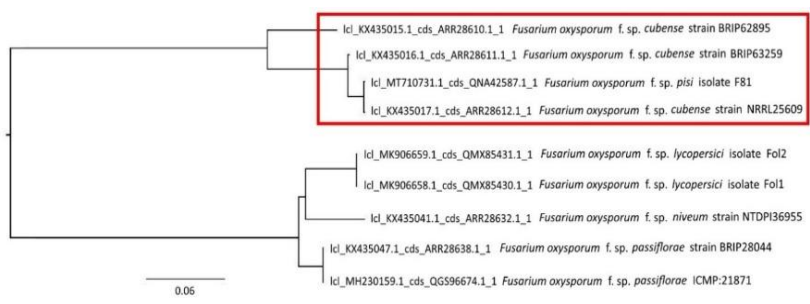

Fig. 5: Phylogenetic tree of SIX9 in formae speciales of $F$. oxysporum. The tree was generated using IQ-TREE (iqtree.cibiv.univie.ac.at)

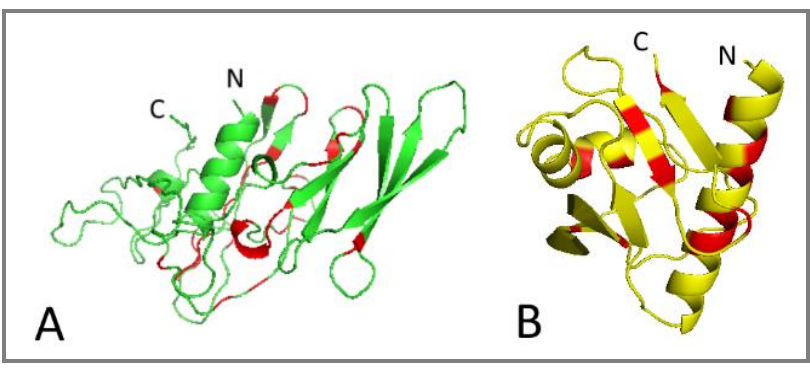

Fig. 6: Putative 3D structure of SIX6 (A) and SIX9 (B) of Foc. SIX6 and SIX9 of Foc are small proteins with the size of 217 and 118 amino acids, respectively. The structures were generated using trRosetta software (https://yanglab.nankai.edu.cn/trRosetta/). Polymorphisms of SIX6 and SIX9 in Foc were plotted in red colour using PyMOL. The $\mathrm{C}$ - and $\mathrm{N}$-terminus were indicated

however, has 6 polymorphic residues in the signal peptide whilst the rest are scattered.

\section{SIX6 of Foc is highly diverse compared to other formae speciales based on the rate of synonymous mutation}

Polymorphisms in SIX6 and SIX9 of all formae speciales were observed at the amino acid level using Selecton analysis (Fig. 7). We found that SIX6 is more diverse compared to SIX9 with 60 residues with a sign of positive

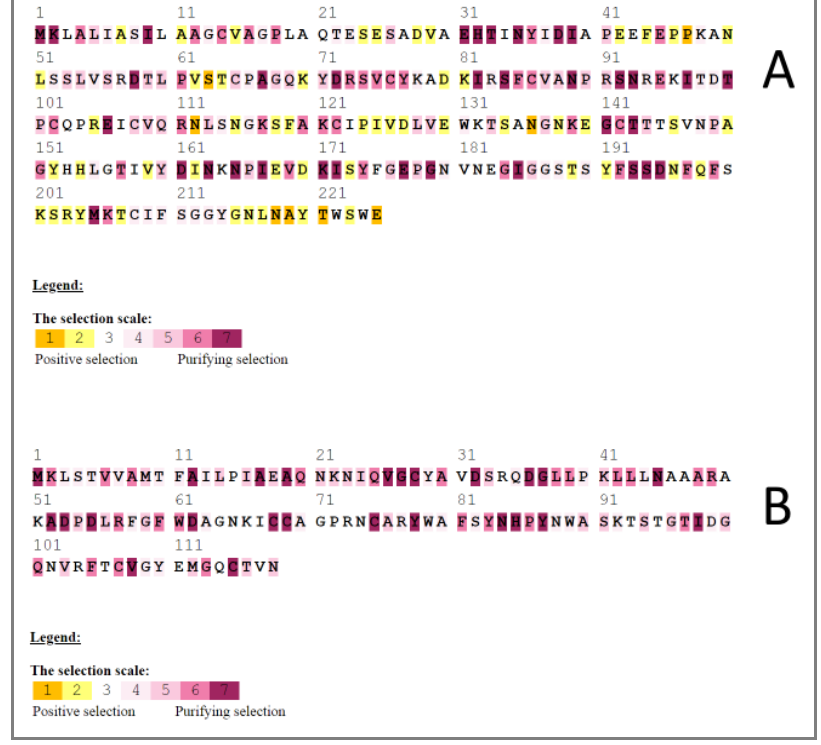

Fig. 7: Site-specific positive selection and purifying selection of SIX6 (A) and SIX9 (B) proteins. Positive selection (orange, level 1) indicates high level for polymorphisms whereas purifying selection (purple, level 7) indicates low level for polymorphisms

selection (yellow to orange scale), comprising $26.67 \%$ of the length of the protein (Fig. 7A). Conversely, SIX9 did not show any sign of positive selection (Fig. 7B). We further studied the distribution of $\mathrm{Ka}$ and $\mathrm{Ks}$ values by conducting a pairwise comparison between different SIX6 and SIX9 sequences using SNAP. The distribution of $\mathrm{Ka}$ and $\mathrm{Ks}$ values between SIX6 sequences from different formae speciales indicates that SIX6 underwent a purifying selection where the rate of $\mathrm{Ks}$ is higher than the rate of $\mathrm{Ka}$ between all the sequences of SIX6 in the alignment (Fig. 8A). Interestingly, the majority of the high $\mathrm{Ka}$ and $\mathrm{Ks}$ values observed in the distribution were contributed by the two Foc SIX6 sequences (KX435008.1 and KX435007.1). The exclusion of the two Foc SIX6 sequences resulted in the change in the distribution of the $\mathrm{Ka}$ and $\mathrm{Ks}$ values (Fig. 8B). This would indicate that in the case of the SIX6 gene, there is a high degree of diversity between Foc and other formae speciales.

We also performed a similar pairwise analysis of $\mathrm{Ka}$ and Ks with SIX9 sequences and we observed a similar distribution of $\mathrm{Ka}$ and $\mathrm{Ks}$, which also suggests that purifying selection was acted upon the $S I X 9$ gene. However, exclusion of the three Foc SIX9 sequences (KX435015.1, KX435016.1 and KX435017.1) did not produce a significant difference in the distribution of $K a$ and $K s$, which suggests that the diversity of the SIX9 gene between different formae speciales is relatively low (Fig. 9).

\section{Discussion}

Two effector candidates from $F$. oxysporum were investigated in this study, SIX6 and SIX9. Both genes showed expressions that are specific only in the roots and 


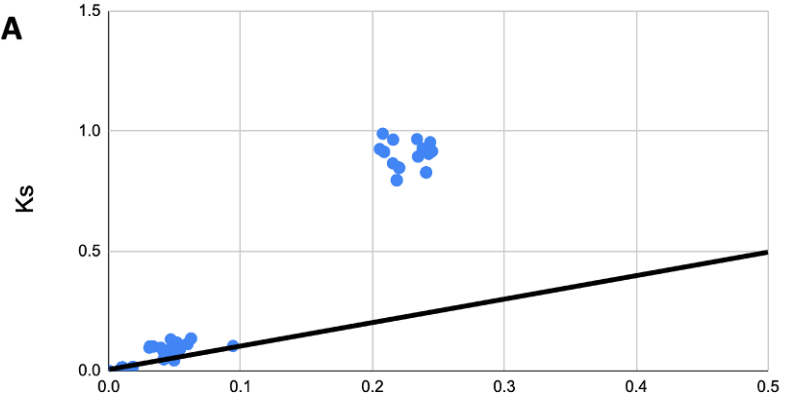

B

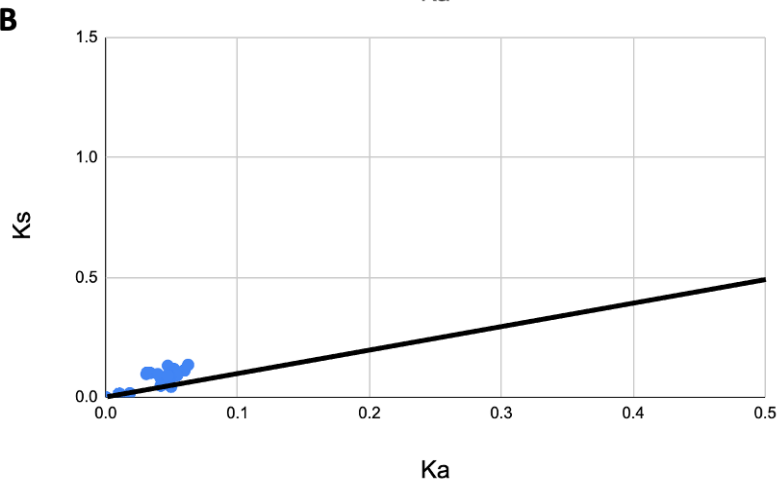

Fig. 8: Distribution of synonymous (Ks/dS) and nonsynonymous $(\mathrm{Ka} / \mathrm{dN})$ substitution rate across different SIX6 sequences. Displayed are the distribution of $\mathrm{Ka}$ and $\mathrm{Ks}$ with two sequences of Foc SIX6, KX435008.1 and KX435007.1, included (A) or excluded (B) from pairwise analysis of $\mathrm{Ka}$ and Ks. Black line represents $\mathrm{Ks}=\mathrm{Ka}$

happened at the earliest stage of infection peaking at $6 \mathrm{dpi}$ and 9 dpi for $S I X 6$ and $S I X 9$, respectively. The difference in the expression peak between SIX6 and SIX9 would indicate the different stages in which each gene plays its part during the infection of the host. Both SIX6 and SIX9 exhibited a degree of conservation across different formae speciales of $F$. oxysporum infecting a wide range of hosts. We also observed several features that support the hypothesis that SIX6 and SIX9 are effectors by the existence of signal peptide at the $\mathrm{N}$ terminal of the protein sequence of both proteins, along with the high number of conserved cysteine residues.

Based on the calculated pairwise Ka and Ks values we observed that both SIX6 and SIX9 genes were under purifying selection. However, based on the distribution of $\mathrm{Ka}$ and Ks values, the diversity between $F o c$ and other formae speciales is higher in SIX6 compared to SIX9. The clustering of the $\mathrm{Ka}$ and $\mathrm{Ks}$ values in the plot mimics the clades in the phylogenetic tree with the two clusterings of the Ka and Ks. The distribution of the Ka and Ks values suggests that both SIX6 and SIX9 underwent purifying selection across the different formae speciales.

The suggestion that SIX6 underwent purifying selection might at first seem to contradict the result from the Selecton analysis where SIX6 was reported to undergo positive selection. This discrepancy can be explained by the
A

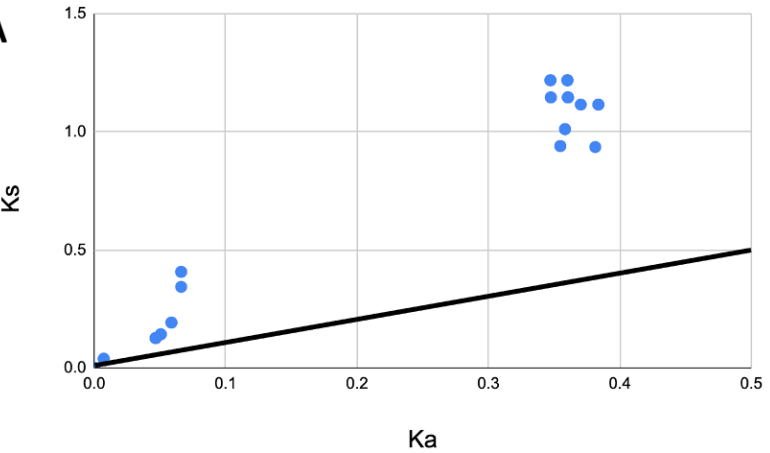

B

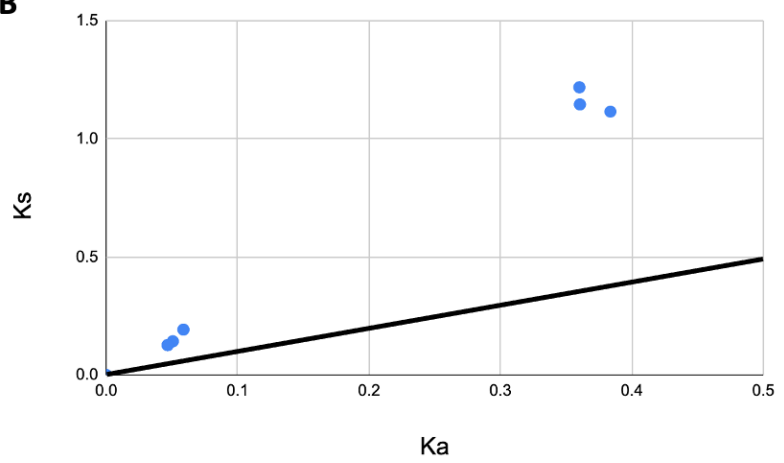

Fig. 9: Distribution of synonymous (Ks/dS) and nonsynonymous $(\mathrm{Ka} / \mathrm{dN})$ substitution rate across different SIX9 sequences. Displayed are the distribution of $\mathrm{Ka}$ and $\mathrm{Ks}$ with three sequences of Foc SIX9, KX435015.1, KX435016.1 and KX435017.1, included (A) or excluded (B) from pairwise analysis of Ka and Ks. Black line represents Ks = Ka

difference in the way $\mathrm{Ka}$ and $\mathrm{Ks}$ were measured between the two methods. In pairwise comparison using SNAP, the $\mathrm{Ka}$ and Ks were measured across all the codon sites within the gene, while in the case of Selecton, Ka and Ks were measured in a codon-by-codon manner. This would allow Selecton to identify sites that are undergoing either positive balancing or purifying selection.

While in general SIX6 gene across different formae speciales is under purifying selection, we observed that the SIX6 gene showed a great degree of diversity between Foc and other formae speciales as shown both by the phylogenetic tree and the distribution of Ka and Ks value. These results in combination with the result from Selecton analysis lead us to believe that in the context of Foc clade, we are observing a degree of relaxation in the purifying selection acting on the gene. Whether the hypothesized relaxation of purifying selection in Foc clade corresponds to the specificity of Foc SIX6 to a certain host is a question that remains to be answered. In the case of SIX9, both the analysis of pairwise $\mathrm{Ka}$ and Ks distribution and Selecton analysis agreed on the possibility of purifying selection acting on the SIX9 gene. The peak of SIX9 gene expression also happened at a later day compared to SIX6 (Fig. 1B), suggesting that $S I X 9$ might be mediating the infection at the later stage of the infection compared to SIX6. 
Based on the difference in the pattern of nucleotide and amino acid diversity between SIX6 and SIX9 when we compared Foc and other formae speciales, it is suggested that the SIX6 gene in Foc have gained a degree of adaptation that is specific to the main host of Foc. The low degree of both nucleotide and amino acid diversity in SIX9 would suggests that the role that it plays during the infection is non-formae speciales specific and conserved across different formae speciales. Further study by disrupting the expression of either the gene, and in the case of FocSIX6, the expression under different formae speciales, would be needed to further dissect the roles of SIX6 and SIX9 as an effector of $F$. oxysporum.

F. oxysporum has attracted plant pathologists across the globe due to its devastating impact on the economy of many countries and also because of its evolutionary quests affecting different hosts, hence the name $F$. oxysporum species complex (FOSC) (Di et al. 2016). The soil-borne fungus in FOSC includes both nonpathogenic and pathogenic strains (Gordon 2017). In banana and many other important crops, the pathogenic strains invade roots and cause wilting via colonization of xylem tissues (Dita et al. 2018). More than 120 formae speciales have been identified in pathogenic Fo strains (Edel-Hermann and Lecomte 2019). The formae speciales refers to narrow host specificity, where each forma specialis infects specific plant species (Gordon 2017). However, this host range was subsequently found to be wider in many formae speciales not only in plants (EdelHermann and Lecomte 2019) but also in humans (Zhang et al. 2020). The Fo pathogenic strains usually are hemibiotrophs, performing a biotrophic lifestyle at early stages of infection and at later stages release toxins in order to kill the host cells and obtain nutrients on the dead tissue (Michielse and Rep 2009; Horbach et al. 2011).

The co-evolutionary arms race between pathogens and hosts can be observed in a interplay between genes involved in the interaction, namely resistance $(R)$ genes in host plants and avirulence $(A v r)$ genes in pathogens (Jones and Dangl 2006). Avr genes are known as effectors that have the ability to manipulate the host immune system to avoid detection and optimizing the virulence function (Presti et al. 2015). Host plants evolved by recognizing these specific proteins via $\mathrm{R}$ genes (Derbyshire 2020). SIX genes have been reported involved in virulence and host manipulations in susceptible cultivars (Rep et al. 2005; Houterman et al. 2009; Widinugraheni et al. 2018; An et al. 2019). However, in resistant cultivars, these genes mediated and triggered resistance (Rep et al. 2004; Houterman et al. 2008, 2009). SIX6 was reported to contribute to the virulence of $\mathrm{Fol}$ and suppresses I-2-mediated cell death (Gawehns et al. 2014). However, its role in Foc-banana pathosystem has not been determined yet. To overcome the fungal attack, the host cells were expressing the pathogenesis-related $(P R)$ genes which are crucial components of the plant innate immune system especially systemic acquired resistance, thus extensively utilized as markers for defense signaling pathways (Ali et al. 2018). The over expression of the $P R-1$ gene was reported to enhance resistance in plants during bacterial and fungal attacks (Chandrashekar et al. 2018; Lu and Edwards 2018; Tosarini et al. 2018; Akbudak et al. 2020). PR-3 gene encodes a chitinase that disintegrates chitin in fungal cell walls and inhibits the fungal growth (Takahashi et al. 2016; Chandrashekar et al. 2018). In susceptible banana cultivar, these $P R$ genes were highly expressed in the corms and subsequently in the roots. In this study, we have shown that both $P R-1$ and $P R-3$ are displaying expression patterns that are antagonistic to $S I X 6$ and SIX9 genes despite the spatial difference in which they are expressed. This difference can be attributed to the nature of the effector itself, which can trigger virulence response in tissues other than of the initial site of infection. The underlying molecular mechanism in which PR-1 and PR-3 proteins from the host interact with SIX6 and SIX 9 proteins from the pathogen and trigger virulence is still an open question that remains to be answered.

\section{Conclusion}

We found that in the susceptible banana cultivar, SIX6 and SIX9 of Foc TR4 are highly expressed in roots, but not in corms or the leaves. The host, however, expressed the pathogenesis-related $(P R)$ genes, $P R-1$ and $P R-3$, in corms earlier than in the roots. We also discovered that SIX6 and SIX 9 of Foc are polymorphic compared to other formae speciales. Based on the rate of synonymous mutation, SIX6 of Foc showed an increase in diversity, but insufficient to drive positive selection. Conversely, SIX 9 of Foc showed no divergence in the distribution of the $\mathrm{dN} / \mathrm{dS}$ ratio, indicating purifying selection.

\section{Acknowledgements}

We would like to thank the Banana Group and the Research Center for Nanoscience and Nanotechnology, Institut Teknologi Bandung, for facilities given in conducting the experiments. We also thank the Banana Group and the Research Center for Nanoscience and Nanotechnology, Institut Teknologi Bandung, for facilities given in conducting the experiments.

\section{Funding Source}

This research was funded by the Ministry of Research, Technology and Higher Education of the Republic of Indonesia, contract number: 2/AMD/E1/KP.PTNBH/2020 given to RRE.

\section{Author Contributions}

RRE and NF planned the experiments, reviewed and edited the manuscript, INPA and MBB analyzed the data, NF and $\mathrm{MBB}$ write the manuscript and made illustrations. 


\section{Conflict of Interest}

The authors declare that they have no conflict of interest.

\section{Data Availability}

We hereby declare that all data reported in this paper are available and will be produced on demand.

\section{Ethics Approval}

Not applicable.

\section{References}

Akbudak MA, S Yildiz, E Filiz (2020). Pathogenesis related protein-1 (PR1) genes in tomato (Solanum lycopersicum L.): Bioinformatics analyses and expression profiles in response to drought stress. Genomics 112:4089-4099

Ali S, BA Ganai, AN Kamili, AA Bhat, ZA Mir, JA Bhat, A Tyagi, ST Islam, M Mushtaq, P Yadav, S Rawat, A Grover (2018). Pathogenesis-related proteins and peptides as promising tools for engineering plants with multiple stress tolerance. Microbiol Res 212213:29-37

An B, X Hou, Y Guo, S Zhao, H Luo, C He, Q Wang (2019). The effector SIX8 is required for virulence of Fusarium oxysporum f. spp. cubense tropical race 4 to Cavendish banana. Fung Biol 123:423-430

Bai TT, WB Xie, PP Zhou, ZL Wu, WC Xiao, L Zhou, J Sun, XL Ruan, HP $\mathrm{Li}$ (2013). Transcriptome and expression profile analysis of highly resistant and susceptible banana roots challenged with Fusarium oxysporum f. spp. cubense tropical race 4. PLoS One 8; Article e73945

Chandrashekar N, S Ali, A Grover (2018). Exploring expression patterns of $P R-1, P R-2, P R-3$, and PR-12 like genes in Arabidopsis thaliana upon Alternaria brassicae inoculation. 3 Biotech 8:230-239

Cordeiro MCR, MS Silva, EC Oliveira-Filho, ZJG Miranda, F Góis Aquino, RR Fragoso, J Almeida, LRM Andrade (2008). Optimization of a method of total RNA extraction from Brazilian native plants rich in polyphenols and polysaccharides. In: IX Simpósio nacional sobre o cerrado, pp:12-17. Brazil

Czislowski E, S Fraser-Smith, M Zander, WT O'Neill, RA Meldrum, LTT Tran-Nguyen, J Batley, EAB Aitken (2018). Investigation of the diversity of effector genes in the banana pathogen, Fusarium oxysporum f. spp. cubense, reveals evidence of horizontal gene transfer. Mol Plant Pathol 19:1155-1171

Dalio RJD, J Herlihy, TS Oliveira, JM McDowell, M Machado (2018). Effector Biology in Focus: A primer for computational prediction and functional characterization. Mol Plant Microb Interact 31:22-23

Derbyshire MC (2020). Bioinformatic detection of positive selection pressure in plant pathogens: The neutral theory of molecular sequence evolution in action. Front Microbiol 11; Article 644

Di X, FLW Takken, N Tintor (2016). How phytohormones shape interactions between plants and the soil-borne fungus Fusarium oxysporum. Front Plant Sci 7:1-9

Dita M, M Barquero, D Heck, ESG Mizubuti, CP Staver (2018). Fusarium wilt of banana: Current knowledge on epidemiology and research needs toward sustainable disease management. Front Plant Sci 9; Article 1468

Dodds PN, M Rafiqi, PH Gan, AR Hardham, DA Jones, JG Ellis (2009). Effectors of biotrophic fungi and oomycetes: Pathogenicity factors and triggers of host resistance. New Phytol 183:993-1000

Edel-Hermann V, C Lecomte (2019). Current status of Fusarium oxysporumformae speciales and races. Phytopathology 109:512-530

FAO (2020). The State of Agricultural Commodity Markets. FAO, Rome, Italy. Available at: http://www.fao.org/3/cb0665en/cb0665en.pdf (Accessed 18 March 2021)
Gawehns F, PM Houterman, F AIchou, CB Michielse, M Hijdra, BJ Cornelissen, M Rep, FL Takken (2014). The Fusarium oxysporum effector SIX6 contributes to virulence and suppresses I-2-mediated cell death. Mol Plant Microb Interact 27:336-348

Gordon TR (2017). Fusarium oxysporum and the Fusarium wilt syndrome. Annu Rev Phytopathol 55:23-39

Guo L, L Han, L Yang, H Zeng, D Fan, Y Zhu, Y Feng, G Wang, C Peng, X Jiang, D Zhou, P Ni, C Liang, L Liu, J Wang, C Mao, X Fang, M Peng, J Huang (2014). Genome and transcriptome analysis of the fungal pathogen Fusarium oxysporum f. spp. cubense causing banana vascular wilt disease. PLoS One 9; Article e95543

Horbach R, AR Navarro-Quesadac, W Knoggec, HB Deising (2011). When and how to kill a plant cell: Infection strategies of plant pathogenic fungi. J Plant Physiol 168:51-62

Houterman PM, L Ma, GV Ooijen, MJD Vroomen, BJ Cornelissen, FL Takken, M Rep (2009). The effector protein Avr2 of the xylemcolonizing fungus Fusarium oxysporum activates the tomato resistance protein I-2 intracellularly. Plant J 58:970-978

Houterman PM, BJ Cornelissen, M Rep (2008). Suppression of plant resistance gene-based immunity by a fungal effector. PLoS Pathog 4; Article e1000061

Houterman PM, D Speijer, HL Dekker, CGDE Koster, BJ Cornelissen, M Rep (2007). The mixed xylem sap proteome of Fusarium oxysporum-infected tomato plants. Mol Plant Pathol 8:215-221

Jones JDG, JL Dangl (2006). The plant immune system. Nature 444:323-329

Korber B (2000). HIV signature and sequence variation analysis. In: Computational and Evolutionary Analysis of HIV Molecular Sequences, pp: 55-72. Rodrigo AG, GH Learn (Eds.). Kluwer Academic Publishers, Dordrecht, The Netherlands

Laurence MH, BA Summerell, ECY Liew (2015). Fusarium oxysporum f. spp. canariensis: evidence for horizontal gene transfer of putative pathogenicity genes. Plant Pathol 64:1068-1075

Li CY, GM Deng, J Yang, A Viljoen, Y Jin, RB Kuang, CW Zuo, ZC Lv, QS Yang, O Sheng, YR Wei, CH Hu, T Dong, GJ Yi (2012). Transcriptome profiling of resistant and susceptible Cavendish banana roots following inoculation with Fusarium oxysporum f. spp. cubense tropical race 4. BMC Genomics 13:374-384

Li J, B Cornelissen, M Rep (2020). Host-specificity factors in plant pathogenic fungi. Fung Genet Biol 144:103447

Li W, X Ge, W Wu, W Wang, Y Hu, Y Mo, D Sun, S Shi, J Xie (2015). Identification of defense-related genes in banana roots infected by Fusarium oxysporum f. spp. Cubense tropical race 4. Euphytica 205:837-849

Livak KJ, TDSchmittgen (2001). Analysis of relative geneexpression datausing real-time quantitative PCR and the $2-^{\Delta \Delta C \mathrm{~T}}$ method. Methods 25:402-408

Lu S, MC Edwards (2018). Molecular characterization and functional analysis of PR-1 like proteins identified from the wheat head blight fungus Fusarium graminearum. Phytopathology 108:510-520

Ma LJ, HC van der Does, KA Borkovich, JJ Coleman, MJ Daboussi, A Di Pietro, M Dufresne, M Freitag, M Grabherr, B Henrissat, PM Houterman, S Kang, WB Shim, C Woloshuk, X Xie, JR Xu, J Antoniw, SE Baker, BH Bluhm, A Breakspear, DW Brown, RAE Butchko, S Chapman, R Coulson, PM Coutinho, EGJ Danchin, A Diener, LR Gale, DM Gardiner, S Goff, KE Hammond-Kosack, K Hilburn, A Hua-Van, W Jonkers, K Kazan, CD Kodira, M Koehrsen, L Kumar, YH Lee, L Li, JM Manners, D Miranda-Saavedra, M Mukherjee, G Park, J Park, SY Park, RH Proctor, A Regev, MC RuizRoldan, D Sain, S Sakthikumar, S Sykes, DC Schwartz, BG Turgeon, I Wapinski, O Yoder, S Young, Q Zeng, S Zhou, J Galagan, CA Cuomo, HC Kistler, M Rep (2010). Comparative genomics reveals mobile pathogenicity chromosomes in Fusarium. Nature 464:367-373

Mehrabi R, AH Bahkali, KA Abd-Elsalam, M Moslem, SB M'Barek, AM Gohari, MK Jashni, I Stergiopoulos, GHJ Kema, PJGM Wit (2011). Horizontal gene and chromosome transfer in plant pathogenic fungi affecting host range. FEMS Microbiol Rev 35:542-554

Michielse CB, M Rep (2009). Pathogen profile update: Fusarium oxysporum. Mol Plant Pathol 10:311-324

Presti LL, D Lanver, G Schweizer, S Tanaka, L Liang, M Tollot, A Zuccaro, S Reissmann, R Kahmann (2015). Fungal effectors and plant susceptibility. Annu Rev Plant Biol 66:513-545 
Rep M, M Meijer, PM Houterman, HC van der Does, BJ Cornelissen (2005). Fusarium oxysporum evades $I$-3-mediated resistance without altering the matching avirulence gene. Mol Plant Microb Interact 18:15-23

Rep M, HC van der Does, M Meijer, R van Wijk, PM Houterman, HL Dekker, CGD Koster, BJ Cornelissen (2004). A small, cysteine-rich protein secreted by Fusarium oxysporum during colonization of xylem vessels is required for $\mathrm{I}-3$-mediated resistance in tomato. $\mathrm{Mol}$ Microbiol 53:1373-1383

Sonah H, RK Deshmukh, RR Bélanger (2016). Computational prediction of effector proteins in fungi: opportunities and challenges. Front Plant Sci 7; Article 126

Stergiopoulos I, PJD Wit (2009). Fungal effector proteins. Annu Rev Phytopathol 47:233-263

Sun J, J Zhang, H Fang, L Peng, S Wei, C Li, S Zheng, J Lu (2019). Comparative transcriptome analysis reveals resistance-related genes and pathways in Musa acuminata banana 'Guijiao 9' in response to Fusarium wilt. Plant Physiol Biochem 141:83-94

Sun Y, X Yi, M Peng, H Zeng, D Wang, B Li, Z Tong, L Chang, X Jin, X Wang (2014). Proteomics of Fusarium oxysporum race 1 and race 4 reveals enzymes involved in carbohydrate metabolism and ion transport that might play important roles in banana Fusarium wilt. PLoS One 9; Article e113818

Takahashi M, J Shigeto, S Izumi, K Yoshizato, H Morikawa (2016) Nitration is exclusive to defense-related PR-1, PR-3 and PR-5 proteins in tobacco leaves. Plant Signal Behav 11; Article e1197464
Thatcher LF, DM Gardiner, K Kazan, JM Manners (2012). A highly conserved effector in Fusarium oxysporum is required for full virulence on Arabidopsis. Mol Plant Microb Interact 25:180-190

Tosarini TR, PZ Ramos, GS Profeta, RM Baroni, KB Massirer, RM Counago, JMC Mondego (2018). Cloning, expression and purification of kinase domains of cacao PR-1 receptor-like kinases. Protein Expr Purif 146:78-84

Wang S, PC Boevink, L Welsh, R Zhang, SC Whisson, PRJ Birch (2017). Delivery of cytoplasmic and apoplastic effectors from Phytophthora infestans haustoria by distinct secretion pathways. New Phytol 216:205-215

Widinugraheni S, J Nino-Sanchez, HCD Does, PV Dam, FA GarciaBastidas, S Subandiyah, HJG Meijer, HC Kistler, GHJ Kema, M Rep (2018). A SIX1 homolog in Fusarium oxysporum f. spp. cubense tropical race 4 contributes to virulence towards Cavendish banana. PLoS One 13; Article e0205896

Zhang L, A Cenci, M Rouard, D Zhang, Y Wang, W Tang, SJ Zheng (2019). Transcriptomic analysis of resistant and susceptible banana corms in response to infection by Fusarium oxysporum f. spp. cubense tropical race 4. Sci Rep 9; Article 8199

Zhang Y, H Yang, D Turra, S Zhou, DH Ayhan, GA DeIulio, L Guo, K Broz, N Wiederhold, JJ Coleman, K O' Donnell, I Youngster, AJ McAdam, S Savinov, T Shea, S Young, Q Zeng, M Rep, E Pearlman, DC Schwartz, AD Pietro, HC Kistler, LJ Ma (2020). The genome of opportunistic fungal pathogen Fusarium oxysporum carries a unique set of lineage-specific chromosomes. Commun Biol 3; Article 50 